\title{
Macrocausality, Unitarity and Discontinuity Formulae in $S$-Matrix Theory
}

\author{
by \\ D. IAGOLNITZER*
}

\section{Introduction}

The purpose of this lecture is to present the general physical-region properties of multiparticle scattering amplitudes in relativistic quantum theory. The emphasis will be on recent developments. S-matrix theory has been mainly developed for systems of massive particles with shortrange interactions (i.e. the strong interactions) and we only consider here this case.

The key notion which has appeared to be of basic importance, at the begining of the sixties, in the study of collision amplitudes is that of analyticity. In this lecture, I am concerned only with momentum-space analyticity properties in the physical region. (The physical region of a process is the real mass-shell region associated with this process. Precise definitions will be recalled later). Besides its own interest, the knowledge of the physical region structure of multiparticle amplitudes will give precise indications on the general analyticity properties which can be expected away from the physical region, on the "complex mass-shell", as explained in the lectures by Professor Stapp.

Historically, the derivation of analyticity properties has been based in the first part of the sixties on the idea that "the scattering functions should have the maximal analyticity consistent with unitarity". With regard to the physical-region, the program is then to determine the analytic structure of the S-matric from unitarity and a certain " $i \varepsilon$ postulate". Although this approach still keeps its own interest, it has not been possible so far to establish S-matrix theory on firm and precise bases in this

Received August 30, 1976.

* Service de Physique Théorique, Centre d'Etudes Nucléaire de Saclay, BP. n² 2, 91190 Gif-sur-Yvette, France. 
framework, and we are going here to present the alternative framework, developed more recently, which starts, besides unitarity, from the physical principle of macroscopic causality, or macrocausality.

The S-matrix is introduced in section 1, in which general properties, such as unitarity and energy momentum conservation are briefly reviewed.

Macrocausality is then described in section 2, where we shall see that it directly provides a basic essential support, or singular spectrum property of multiparticle amplitudes. This property ensures in particular the existence, for each given physical process, of a unique analytic function, defined in a domain of the complex mass-shell, to which the physicalregion (connected) $S$-matrix is equal at all points which do not lie on $+\alpha$-Landau surfaces, and from which it is a boundary value, from certain "plus $i \varepsilon$ " directions, at almost all $+\alpha$-Landau points. (These analytic functions are those which will be later analytically continued away from the physical region, on the complex mass-shell: see Stapp's lectures).

A second important step in the development of the theory is then the derivation from unitarity of discontinuity formulae around the $+\alpha$ Landau surfaces. These formulae are described in section 4a) and a somewhat more general form in terms of essential support is briefly outlined in section $4 \mathrm{~b})$. They give information on the nature of the $+\alpha$-Landau singularities and also account, as indicated in section 4c), for the macroscopic space-time description of processes. Their derivation is based on a preliminary result, presented in section 3, which follows from macrocausality and unitarity, and gives information on the essential support, or singular spectrum, of the "bubble diagrams functions" which arise in equations derived from unitary.

To achieve the proof of the discontinuity formulae it has been necessary so far, in general, to use, besides macrocausality and unitarity, an assumption according to which the "mixed- $\alpha$ " singularities of the various bubble diagram functions involved in these latter equations should cancel among themselves (assumption of mixed- $\alpha$ cancellation): see subsection 4a). In a broad framework of S-matrix theory ideas, it is natural to expect such a cancellation and the internal consistency of this assumption has been checked in many cases. On the other hand, if one wishes to establish the discontinuity formulae on the basis only of general physical 
principles, it appears as an ad hoc assumption, which it would be satisfactory to remove. A first step in this direction has been carried out in a work by H. P. Stapp and the present author, in which the simplest of the discontinuity formulae, namely the "pole-factorization theorem", is proved (below the 4-particle threshold) on the basis of macrocausality, unitarity and two weak regularity conjectures, which in contrast to mixed- $\alpha$ cancellation, apply directly to the individual scattering or bubble diagram functions. This work is briefly presented in section 5 .

The general problems which arise in connection with this study are outlined in the conclusion (section 6).

For more complete explanations and details on the contents of this lecture, see [1] and the references quoted therein.

\section{Essential support theory}

From the mathematical view point, the results presented are based essentially on the theory of the essential support which originated (in the analytic sense) in 1968 in a work on macrocausality by Professor Stapp and the present author [2] and was developed in various directions by Professor Bros and the present author: see [3] and the references quoted therein for details.

Being given a distribution $f$ defined on $\mathbb{R}^{N}$ or more generally on a real analytic manifold $\mathcal{M}$, we recall, for the purposes of the present lecture, that the essential support of $f$ at a given point $P$ of $\mathscr{M}$ is the closed cone (with apex at the origin in the cotangent space $T_{P}{ }^{*} \mathscr{M}$ at $P$ to $\mathscr{M}$ ) composed of the "singular directions" of $f$ at $P$ along which the generalized Fourier transfom of $f$ at $P$ does not decrease exponentially (in a well specified sense).

It is known that $f$ is analytic at $P$, resp. is at $P$ the boundary value of an analytic function from the directions of an open cone $\Gamma$, if and only if the essential support of $f$ at $P$ is empty (apart from the origin), resp. is contained in the closed convex salient dual cone $C$ of $\Gamma$. More generally, decomposition theorems allow one to write $f$ as a sum of distributions $f_{j}$ which are boundary value of analytic functions $f_{j}$, at $P$ or over real domains $\Omega$ of $\mathscr{M}$. (In this latter case, the directions from which the boundary values are obtained will depend in general on the real point 
$P$ of $\Omega$ ). Finally, we note that results on products of distributions, integrals, etc... are also directly proved in terms of essential supports.

In view of a similar equivalence with analyticity properties, the singular spectrum [4] of a distribution is necessarily contained in its essential support. (The boundary values $f_{j}$ involved in singular spectrum theory may be general hyperfunctions, and not distributions, even if $f$ is a distribution). It is moreover proved by Professor Bony in his lecture that the two notions do coincide. Hence, all results obtained can be equivalently stated in terms of singular spectrum and this leads to hopes of further developments in connection with holonomy theory: see the lectures by Professors Sato and Kawai. The framework of essential support theory remains however the best adapted and the most direct for the results that we discuss here: this is because causality, or other related properties (see section 2 and subsection 4c)) are physically naturally expressed in the form of exponential fall-off properties in space-time, and hence, as we shall see, in terms of essential support properties.

\section{§ 1. General Properties of the S-matrix}

Details on this section can be found in chapter I of [1] and references quoted there. For simplicity we consider here a theory with only one type of particle, a "boson" of mass $m$ and spin zero. All results of section 1 to 4 are however stated in a form whch is fully valid (with only straightforward modifications) in the more general case.

It is assumed in the theory that before and after interaction the physical systems under consideration are represented by vectors of a Hibert space $\mathcal{H}=\underset{q=1,2,3 \ldots}{\oplus} \mathcal{H}_{q}$ of free particle states, where $\oplus$ denotes a direct sum of Hilbert spaces $\mathcal{H}_{q}$ of $q$ particles, each of which is in turn the symmetrized tensorial product of $q$ spaces $\mathscr{H}_{1}$. Finally, $\mathscr{H}_{1}$ is the space of the functions $\phi$ of an energy-momentum on-mass-shall 4 -vector variable $p=\left(p_{0}, \vec{p}\right), p^{2}=p_{0}{ }^{2}-\vec{p}^{2}=m^{2}, p_{0}>0$, which are square integrable with respect to the invariant measure

$$
\delta\left(p^{2}-m^{2}\right) \theta\left(p_{0}\right) d^{4} p=\frac{d^{3} \vec{p}}{2 \sqrt{\vec{p}^{2}+m^{2}}}
$$

It follows from general quantum principles that there exists a linear, 
unitary operator $S$ from $\mathcal{H}$ to $\mathcal{H}$ such that $\left|\left\langle\psi_{2}|S| \psi_{1}\right\rangle\right|^{2}$ is the "transition probability" from an initial free particle state (before interaction) represented by a unit-norm vector $\left|\psi_{1}\right\rangle \in \mathcal{H}$, to a final free particle state (after interaction) represented by a unit-norm vector $\left|\psi_{2}\right\rangle$. (i.e. $\left|\left\langle\psi_{2}|S| \psi_{1}\right\rangle\right|^{2}$ is the probability of detecting $\left|\psi_{2}\right\rangle$ after interaction if the system is represented before interaction by $\left.\left|\psi_{1}\right\rangle \mid\right)$.

Unitarity (which includes linearity) can be derived from a principle of "conservation of probabilities" that we shall not discuss here.

The operator $S$ induces corresponding functionals $S_{m, n}$ which act on pairs $f_{m}, g_{n}, f_{m} \in \mathcal{H}_{m}, g_{n} \in \mathcal{H}_{n}$ and are linear with respect to $f_{m}$ and $g_{n}$. It follows from the probabilistic interpretation of the $S$-matrix that those functionals are bounded in $L^{2}$-norm with respect to $f_{m}$ and $g_{n}$ and hence are in particular tempered distributions on the space of the $m+n$ on-massshell initial and final 4 -momentum variables $p_{k}\left(p_{k}{ }^{2}=m^{2}, p_{k_{0}}>0, \forall k\right)$. A first general property of the $S$-matrix is then energy-momentum conservation, which can be derived for instance from Poincaré invariance of transition probabilities, and can be written, if the 4 -vectors $p_{k}$ are not all parallel, in the form:

$$
S_{m, n}\left(p_{1} \cdots p_{m} ; p_{m+1}, \cdots p_{m+n}\right)=s_{m, n} \times \delta^{4}\left(\sum p_{i}-\sum p_{j}\right)
$$

where the sums $\sum$ run over the initial and final 4-momenta respectively, and $s_{m, n}$ is a tempered distribution defined on the space $\mathscr{M}$ of all initial and final 4 -vectors $p_{k}$ such that $p_{k}{ }^{2}=m^{2}, p_{k_{0}}>0, \forall k$ and $\sum p_{i}=\sum p_{j} ; \mathcal{M}$ is called the physical region of the process $m \rightarrow n$.

In view of stability requirements, $S_{m, n}=0$ if $m=1, n>1$ or $m>1$, $n=1$. On the other hand $S_{1,1}$ is the kernel of the identity operator $S_{1,1}(p ; q)=2 \sqrt{\vec{p}^{2}+m^{2}} \delta^{3}(\vec{p}-\vec{q})$.

For reasons which will appear later, it is finally useful to associate with the family of functionals $S_{m, n}$ a corresponding family of functionals $S_{m, n}^{c}$ called their connected parts. They are defined by induction in a way such that each $S_{m, n}$ is a sum, over all possible partitions of the sets of initial and final particles, of the symmetrized tensorial products of the corresponding connected functionals. These relations are usually written in a diagrammatical form on the momentum-space kernels: 


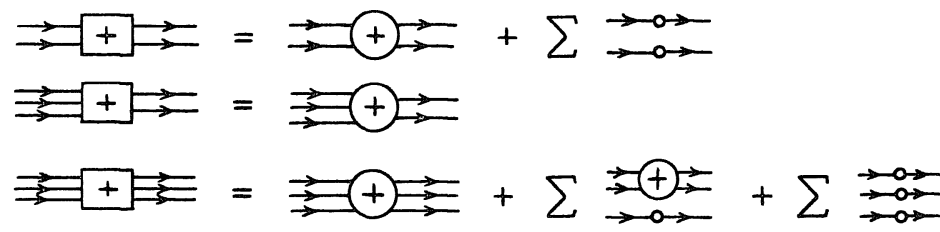

etc...

where connected kernels of the $S$-matrix. The sums $\sum$ refer to the various ways of assigning sets of initial and final 4-momenta to each bubble.

It is easily checked by induction that $S_{m, n}^{c}$ can also be written in the same form as (1) (if all $p_{k}$ are not all parallel):

$$
S_{m, n}^{c}\left(p_{1} \cdots p_{m, n}\right)=f_{m, n}\left(p_{1} \cdot p_{m+n}\right) \times \delta^{4}\left(\sum p_{i}-\sum p_{j}\right)
$$

where $f_{m, n}$ is again a tempered distribution, defined on the physical region of the process $m \rightarrow n$, and is called the scattering function of this process.

\section{§ 2. Macrocausality and Essential support of Scattering Functions ${ }^{[5]}$}

a) Preliminary definitions.

Being given a process $m \rightarrow n$ and the corresponding physical-region $\mathscr{M}$, the cotangent space $T_{p} * \mathscr{M}$ to $\mathscr{M}$ at point $P=\left\{p_{k}\right\} \in \mathscr{M}$ is the quotient space $\mathbb{R}^{4(m+n)} / N(P)$ of $\mathbb{R}^{4(m+n)}$ by the conormal space at $P$ to $\mathscr{M}$. The scalar product $p \cdot u$ of a point $p=\left\{p_{k}\right\} \subset \boldsymbol{R}^{4(m+n)}$ with a point $u=\left\{u_{k}\right\}$ in the dual space is defined by convention as:

$$
p \cdot u=\sum_{i \in I} p_{i} \cdot u_{i}-\sum_{j \in J} p_{j} \cdot u_{j} ; p_{k} \cdot u_{k}=p_{k_{0}} u_{k_{0}}-\vec{p}_{k} \cdot \vec{u}_{k}
$$

where the sums $\sum$ run over the sets I,J of initial and final particles respectively. The space $N(P)$ is, in view of the definition of $\mathscr{M}$ in $\boldsymbol{R}^{4(m+n)}$, the space of vectors $n=\left\{n_{k}\right\} \in \mathbb{R}^{4(m+n)}$ of the form $n_{k}=\lambda_{k} P_{k}+a$, $\forall k$, where $\lambda_{k}$ is an arbitrary real scalar and a is a 4 -vector independent of $k$.

Being given a point $P=\left\{P_{k}\right\} \in \mathcal{M}$ and a point $u=\left\{u_{k}\right\} \in \boldsymbol{R}^{4(m+n)}$, it is useful to consider the set of $m+n$ "trajectories" in 4-dimensional spacetime, which are for each $k$ the lines passing through $u_{k}$ and parallel to 
$P_{k}$. It is clear that the relative configuration of these trajectories does not change if $u$ replaced by $u+n, n \in N(P)$. A point in $T_{p} * \mathcal{M}$ is then uniquely characterized by a relative configuration in space-time of $m+n$ line respectively parallel to the 4 -vectors $P_{k}$.

A classical multiple scattering diagram $\mathscr{D}$ in space-time is a diagram with oriented external and internal lines. Each external line is either initial or final. Each initial (resp. final) line $k$ ends at (resp. is issued from) a given space-time vertex $v_{k}$. Each internal line issues from a vertex $\left(v_{l}\right)_{i n}$ and ends at another vertex $\left(v_{l}\right)_{f}$ (which is different from $\left(v_{l}\right)_{i n}$ in space-time). Finally, each external or internal line possesses a 4 -momentum $p_{k}$, or $p_{l}$ and the following classical laws must be satisfied:

i) mass-shell constraints on all 4-momenta.

ii) energy-momentum conservation at each vertex $v$.

iii) each line is oriented in space-time in the direction of its 4-momentum. With each line $k$, or $l$, is associated its "trajectory" which is the full straight line in space-time which is parallel to $p_{k}$, resp. $p_{l}$, and passes through $v_{k}$, resp. through $\left(v_{l}\right)_{i n}$ and $\left(v_{l}\right)_{f}$. In the case when all incoming and outgoing 4-momenta involved at a vertex $v$ are parallel, the vertex $v$ is allowed to be possibly an infinity in space-time in some direction. All trajectories involved at $v$ must then be parallel, but are not required to coincide. A condition of "angular momentum conservation" will however be required as proposed by Professor Stapp, in this case (It is automatically satisfied if $v$ is not at infinity).

A diagram $\mathscr{D}$ associated with a process $m \rightarrow n$ is a diagram, possessing the above properties, with $m$ initial and $n$ final (external) lines. We shall denote by $D$ the topological graph which characterizes its topological structure. Being given a point $P$ in $\mathscr{M}, P$ is said to belong to the $+\alpha$ - Landau surface $L(D+)$, if there exists a diagram $\mathscr{D}$ whose topological structure is $D$ and whose set of external 4-momenta is $P$.

\section{b) Macrocausality.}

Macroscopic causality is an expression of the physical idea that any energy-momentum transfer over macroscopic distances that cannot be attributed to stable physical particles in accordance with classical ideas gives effects that are damped exponentially with distance (short range of the 
interactions).

More precisely, it is useful to consider for each initial of final particle $k$ a family of possible "wave functions" (see section 1) of the form:

$$
\left(\phi_{k}\right)_{\tau, u_{k}, P_{k}}(p)=\chi_{k}(p) e^{-i \tau u_{k} \cdot p} e^{-\tau \tau \Phi\left(p ; P_{k}\right)}
$$

where $P_{k}$ is an on mass-shell 4 -vector, $u_{k}$ is a space-time 4 -vector, $\tau$ and $\gamma$ are positive scalars, $\chi_{k}$ is locally analytic at $P_{k}$ (and has at most slow increase at infinity), and $\Phi$ is for instance of the form $\left(\vec{p}-\vec{P}_{k}\right)^{2}+\lambda\left(p_{0}\right.$ $\left.-P_{k_{0}}\right)^{2}, \lambda \geqq 0$ (i.e. has the properties of the functions $\Phi$ of essential support theory).

The multiplication by $e^{-i \tau u_{k} \cdot p}$ corresponds to a space-time translation of the state by the 4 -vector $\tau u_{k}$.

Although quantum states are in general very different from point particles of classical physics, it turns out [5] that, in the limit when $\tau \rightarrow \infty$, the free particle state whose wave function is given by (7) is asymptotically localized, up to exponential fall-off with $\tau$, around a classical trajectory. More precisely, it is localized in momentum-space around the 4-momentum $P_{k}$, and in a space-time coordinate system scaled to $\tau$, around the trajectory $\left(P_{k}, u_{k}\right)$ (i.e. parallel to $P_{k}$ and passing through $\left.u_{k}\right)$.

Macrocausality is then first expressed as a certain exponential fall-off property, when $\tau \rightarrow \infty$, of the transition probability $W$ between the displaced initial and final particles whose wave functions are given by (7) if $(P, u)$ is not causal $\left(P=\left\{P_{k}\right\}, u=\left\{u_{k}\right\}\right)$, i.e. if there is no diagram $\mathscr{D}$ whose external trajectories are $\left(P_{k}, u_{k}\right)$-(or in exceptional cases, if $(P, u)$ is not a limit of such points). Exponential fall-off follows physically on the one hand from the above mentioned localization properties of the particles involved, and on the otherhand from the "short range" of interactions.

If $(P, u)$ is causal, but corresponds only to diagrams $\mathscr{D}$ composed of several disconnected parts linking together respectively the particles of various subgroups $K$, than the same exponential fall-off property is now assumed (for the same physical reasons) to hold for $W-\prod_{K} W_{K}$, where each $W_{K}$ is the transition probability of the process whose initial and final wave functions are those of the subgroup $K$. This factorization 
property can in turn be shown (up to problems, in particular cases, which we shall not discuss here) to be equivalent to an exponential fall-off property of the connected amplitudes $S^{c}\left(\left\{\left(\phi_{k}\right)_{\tau, u_{k}, P_{k}}\right\}\right)$ when $(P, u)$ is not causal, or also when $(P, u)$ is causal, but cannot correspond to a connected $\mathscr{D}$ (composed of only one connected part).

The connected amplitude $S_{m, n}^{c}\left(\left\{\left(\phi_{k}\right)_{\tau, u_{k}, P_{k}}\right\}\right)$ appears to be a generalized Fourier transform of the type introduced in essential support theory and the above mentioned exponential fall-off properties derived from macrocausality can then be expressed in the form:

\section{Essential support property of scattering functions}

"The essential support of a scattering function $f$ at any point $P$ of the physical region $\mathscr{M}$ is contained in the set $C_{+}(P)$ of points $u=\left\{u_{k}\right\}$ (defined modulo $N(P)$ ) such that $(P, u)$ is causal and corresponds at least to one connected diagram $\mathscr{D}^{\prime \prime}$.

This property was postulated independently in terms of singular spectrum by Professors Pham and Sato [6].

The set $C_{+}(P)$ is clearly empty apart from the origin if $P$ does not lie on the $+\alpha$-Landau surface $L\left(D_{+}\right)$of at least one connected graph $D$. These surfaces are known to be analytic condimension 1 submanifolds of $\mathscr{M}$ and are not dense in $\mathscr{M}$. At a $+\alpha$-Landau point $P$, $C_{+}(P)$ is in general composed of only one direction, which is conormal at $P$ to the surface $L\left(D_{+}\right)$which contains $P$, and is oriented towards the "physical side" of this surface (The latter is always well determined by certain convexity properties of the $+\alpha$-Landau surfaces).

If $P$ lies on several $+\alpha$-Landau surfaces $L\left(D_{+}{ }^{\prime}\right), L\left(D_{+}{ }^{\prime \prime}\right), \cdots$ such that $D^{\prime}, D^{\prime \prime}, \cdots$ are all various "contractions" of a common "parent graph" $D$ and if $P$ lies also on the closure of $L\left(D_{+}\right)$, then $C_{+}(P)$ is a closed convex salient cone (with apex at the origin) obtained by positive linear combinations of vectors which lie in the various directions associated as before with each surface $L\left({D_{+}}^{\prime}\right), L\left(D_{+}{ }^{\prime \prime}\right), \cdots$ involved at $P$.

The points $P$ which have not yet been covered are either points which lie in the interaction of several $+\alpha$-Landau surfaces with no "common parent" $\left(C_{+}(P)\right.$ is then the union of the sets associated with 
each graph or parent graph involved at $P$ ), or points $P=\left\{P_{k}\right\}$ such that two or more intial, or two or more final $P_{k}$ are parallel. In this case, the possibility of vertices at infinity mentioned in the definition of the diagrams $\mathscr{D}$, and of corresponding displacements of parallel initial, or parallel final, trajectories, has to be considered in the specification of $C_{+}(P)$.

At the points of these last two classes, $C_{+}(P)$ is no longer in general always contained in a closed convex salient cone. They however belong to low dimension submanifolds of $\mathscr{M}$ and if they are excluded, the basic result announced in the introduction, follows: there is a unique function $f$, analytic in a domain of the complexified manifold $\mathscr{M}$, to which $f$ is equal at all points which do not lie on $+\alpha$-Landau surfaces (of connected graphs) and from which it is a "plus $i \varepsilon$ " boundary value at $+\alpha$ -Landau points (The plus $i \varepsilon$ directions at a point $P$ are those of the open dual cone of $\left.C_{+}(P)\right)$.

\section{§ 3. Essential Support of Bubble Diagram Functions (Structure Theorem) ${ }^{(7)}$}

A bubble diagram function $F_{B}$ is an integral, over internal on massshell 4-momenta, of a product of connected momentum-space kernels of $S$ or $S^{-1}=S^{\dagger}$, associated with the bubbles of a "bubble diagram" $B$. The bubbles of $B$ are connected by directed lines, which always run from left to right. $F_{B}$ is usually written in the same diagrammatical form as $B$.

\section{Example.}

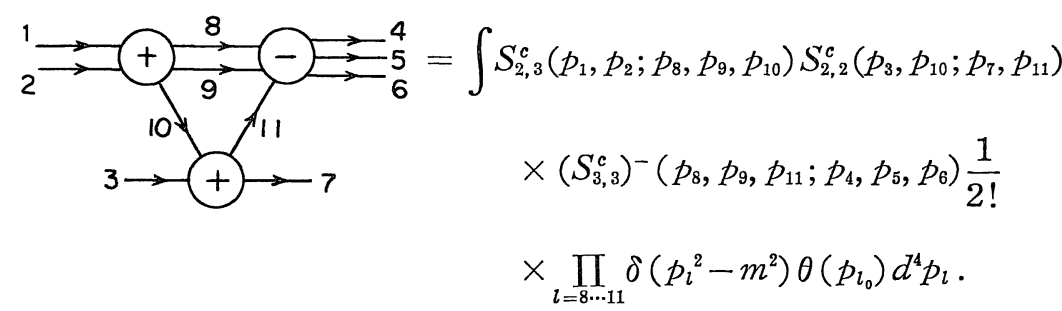

(The factor $1 / 2$ ! associated with the set of two lines 8,9 between two bubbles arises from appropriate conventions).

A bubble diagram function $F_{B}$ can be defined, as $S$ itself, as the 
kernel of a bounded operator in $L^{2}$-norm and is therefore known to be a well defined tempered distribution on the space of all initial and final (external) on mass-shell 4-momenta of $B$. As easily checked, it also satisfies energy-momentum conservation, i.e. one has in the same way as in (5):

$$
F_{B}=f_{B} \delta^{4}\left(\sum p_{i}-\sum p_{j}\right)
$$

A connected graph $D_{B}$ associated with a bubble diagram $B$ is a graph obtained by replacing each bubble $b$ of $B$ by a connected subgraph $D_{b}$ and by moreover attributing $a+\operatorname{sign}$ to each external line of $D_{b}$ if $b$ is $a$ +bubble and $a$-sign to each internal line of $D_{b}$ if $b$ is $a$-bubble. (Each $D_{b}$ is allowed to be a trivial graph, consisting of only one vertex replacing $b$, with no internal lines).

An example of a $D_{B}$ associated with the bubble diagram already mentioned is:

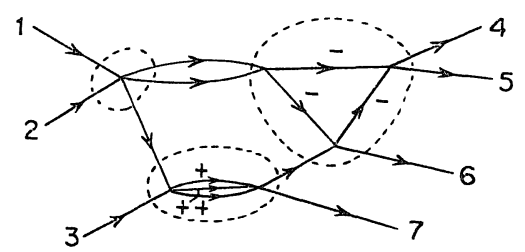

A Space-time representation $\mathscr{D}_{B}$ of $D_{B}$ is a space-time diagram which obeys the same laws than the diagrams $\mathscr{D}$ introduced in section 2 except that property iii) is modified as follows.

iii) Each internal line $l$ of $\mathscr{D}_{B}$ is oriented in the direction of its 4-momentum, resp. in the opposite direction, if $l$ has $a+$ sign, resp. $a$-sign. It is oriented in the direction of its 4 -momentum, or in the opposite direction, or has zero length (i.e. the vertices from which it is issued and to which it ends are represented by the same space-time point) if $l$ is an original internal (unsigned) line of $B$.

A " $u=0$ point $P \in \mathscr{M}$ of $B$ is by definition such that there exists a $\mathscr{D}_{B}$ whose external trajectories have the 4-momenta $P_{k}$ and all meet at a common point in space-time, while at least one internal trajectory does not pass through this point. (This definition is to be slighly completed if all such internal trajectories are associated with internal lines of sub- 
diagrams $\mathscr{D}_{b}$ ).

The following theorem then holds:

\section{Essential support property of bubble diagram functions.}

"If $P$ is not a $u=0$ point, then the essential support of $f_{B}$ at $P$ is contained in the set $C_{B}(P)$ at points $u=\left\{u_{k}\right\}$ (defined modulo $N(P)$ ) which ensure the existence of at least one $\mathscr{D}_{B}$ whose external trajectories are $\left(P_{k}, u_{k}\right)$."

This result is a direct consequence of the general results on products and integrals of distributions of essential support theory (The essential support of $a+$ bubble is given in section 2. The essential support of - bubble is opposite to that of the corresponding + bubble by virtue of unitarity). At $u=0$ points $P$, no information is obtained. These points correspond in fact to situations when the general theorems on products of distributions cannot be applied, and all directions in $T_{p} * \mathcal{M}$ may a priori be singular at $P$. However, we shall see in section 5 that it is necessary to have information at these points in order to derive discontinuity formulae without ad hoc assumptions of mixed- $\alpha$ cancellation. In fact all points $P \in \mathscr{M}$ are $u=0$ points for certain bubble diagram functions involved there, such as $F_{B}=+0$. If $D_{B}$ is the graph

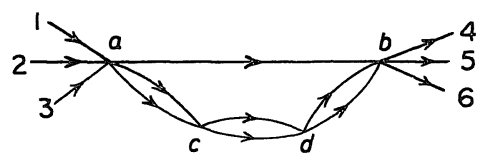

and if $A, B, C, D$ denote the space-time points representing $a, b, c, d$ in a corresponding $\mathscr{D}_{B}$, then, for any given values of the external 4 -momenta, a $\mathscr{D}_{B}$ can always be constructed with $A \equiv B$ on the one hand, $C$ and $D$ at infinity on the other hand:there always exist on mass-shell 4 -vectors $k_{1}, k_{2}, k_{3}$ such that $k_{1}=k_{2}, k_{1}+k_{2}+k_{3}=p_{1}+p_{2}+p_{3}$. The trajectories of the two lines from $a$ to $c$, resp. of the two lines from $d$ to $b$, are parallel lines in space-time, oriented in the direction of $k_{1}=k_{2}$, resp. in the opposite direction, and passing through $A=B$. Since $C$ and $D$ are at infinity, the trajectories of the two internal original lines of $B$ from $c$ to $d$, which must also be parallel to $k_{1}=k_{2}$, do not need however to pass through $A=B$. 
For the purposes of section 5, we shall assume a general regularity property which seems reasonable, but goes beyond macrocausality. A $u=0$ point $P$ will be said to be of the first type if all internal trajectories of corresponding diagrams $\mathscr{D}_{B}$ which do not pass through the meeting point of the external trajectories are associated, as in the example given above, with original internal lines of $B$ and join vertices which are at infinity. We then state:

Regularity conjeciure at (first type) $u=0$ points

"The essential support of $f_{B}$ at a (first type) $u=0$ point $P$ is contained, as before, in the set $C_{B}(P)$ ".

We note that, in view of the definitions, the consideration of limiting procedures does not change this conjecture. By limiting procedures, we mean the replacement of $C_{B}(P)$ by the fiber at $P$ of the closure $\bar{C}_{B}$ of $C_{B}=\bigcup_{p}\left(p, C_{B}(p)\right)$ in $T^{*} \mathscr{M}$, or more refined procedures, such as those proposed in the lecture by Professors Stapp and Kawai, in which modified diagrams $\mathscr{D}_{B}$ are considered (The 4-momenta are no longer required to be on mass-shell, although they must tend to on shell values in the limit). A regularity conjecture covering all $u=0$ points is presented in their lecture. These points seem however more exceptional and we shall not need this conjecture here.

\section{§4. Discontinuity Formulae and Space-Time Description of Processes}

a) General derivation of discontinuity formulae [8]

We consider in this section a given process $m \rightarrow n$, its physical region $\mathscr{M}$ and its scattering function $f$, and for simplicity we first consider a point $P \in \mathscr{M}$ which lies on only one $+\alpha$-Landau surface $L\left(D_{+}\right)$, associated with a given (connected) graph $D$. To be more precise it is assumed moreover that $P$ does not lie in the closure of any other $+\alpha$ -Landau surface and that the initial, resp. the final, 4-momenta $P_{k}$ cannot be parallel.

The surface $L\left(D_{+}\right)$then divides $\mathcal{M}$ locally into two parts, one of 
which is the "physical side" of this surface (and is, as already mentioned, well characterized by certain convexity properties).

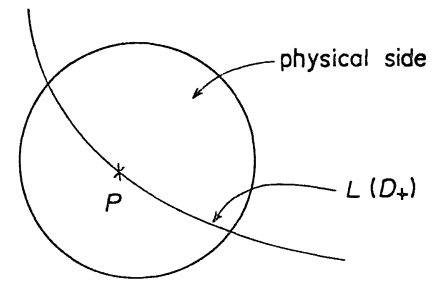

From the result of section 2, the essential support of $f$ at $P$ is composed of only one direction and $f$ is known to be at $P$ the boundary value of the analytic function $f$ associated with the process considered, from "plus $i \varepsilon$ " directions. Moreover, $f$ is known to be locally analytic on each (real) side of $L\left(D_{+}\right)$.

Algebraic manipulations of equations of the form $S S^{-1}=1, \cdots$ are then used (see illustration in section 5) to write $f$ locally as a sum of two terms:

$$
f=d+r
$$

which have the following properties:

i) $\quad d=0$ on the non physical side of $L\left(D_{+}\right)$

ii) $r$ is a sum of bubble diagram functions $f_{B}$, associated with various types of bubble diagrams $B$, which have all a common property:there exists no $\mathscr{D}_{B}$ coinciding with the causal $\mathscr{D}$ associated at $P$ with $D$ (up to dilation or space-time translation), or even coinciding with it after removing zero length lines.

Under the conditions mentioned above on $P$ ( $P$ lies on no other $+\alpha$-Landau surface, $\cdots$ ), property ii) ensures that there is no $\mathscr{D}_{B}$ with only + or zero internal lines (i.e. lines oriented in the direction of their 4-momentum, or with zero length) whose external lines have the 4-momenta $P_{k}$.

On the other hand, there exist in general many possible graphs $D_{B}$ and space-time representations $\mathscr{D}_{B}$ at $P$, associated with the above $f_{B}$, which include + , - or zero lines, or-and zero lines. Under the conditions on $P$, it is easily checked that in the latter case ( - and zero lines), the only possible singular direction of $f_{B}$ at $P$ is the direction 
$C_{-}(P)$, opposite to the direction $C_{+}(P)$. Then, if one just ignores, as a consequence of mixed- $\alpha$ cancellation assumptions, the remaining possibility of $\mathscr{D}_{B}$ including both + and - (and possibly zero) lines (mixed $\alpha$ Landau diagrams), one concludes that the essential support of $r$ at $P$ is (contained in) $C_{-}(P)$. As a consequence, $r$ is at $P$ the "minus $i \varepsilon$ " boundary value of an analytic function $\boldsymbol{r}$ (The minus is directions are those of the open dual cone to $C_{-}(P)$ and are opposite to the $i \varepsilon$ ones).

Since $d=0$ on the non physical side of $L\left(D_{+}\right)$(see property $i$ )) $f=d$ there; hence $\boldsymbol{r}$ is a "minus $i \varepsilon$ " analytic continuation of $f$ around $L\left(D_{+}\right)$, and $d=f-r$ appears explicitly as the discontinuity of $f$ across $L\left(D_{+}\right)$, i.e. as the difference between the boundary values of its plus $i \varepsilon$ and minus $i \varepsilon$ analytic continuations around $L\left(D_{+}\right)$.

Let us describe the results obtained:

\section{Discontinuity formulae}

If $D$ is a graph with no set of more than one line between two vertices, $d \times \delta^{4}\left(\sum p_{i}-\sum p_{j}\right)$ is the bubble diagram function obtained by replacing all vertices of $D$ by + bubbles. For instance, if

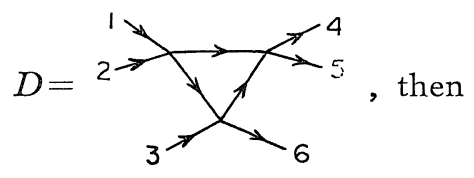

$$
\begin{aligned}
d \times \delta^{4}\left(\sum p_{i}-\sum p_{j}\right)= & \\
\equiv & \int S_{2,2}^{c}\left(p_{1}, p_{2} ; p_{7}, p_{8}\right) S_{2,2}^{c}\left(p_{8}, p_{3} ; p_{6}, p_{9}\right) \\
& \times S_{2,2}^{c}\left(p_{7}, p_{9} ; p_{4}, p_{5}\right) \prod_{l=7,8,9} \delta\left(p_{l}{ }^{2}-m^{2}\right) \theta\left(\left(p_{l}\right)_{0}\right) d^{4} p_{l}
\end{aligned}
$$

If the graph $D$ involves sets of more than one line between two vertices, for instance $D={ }_{4}^{3}$, the formula is to be slightly modified. We shall not discuss this here. (In this latter case, the derivation makes use in general so far of algebraic manipulations involving infinite series, rather than finite number of terms, and is therefore not fully rigorous). 
We conclude with some remarks:

i) In a number of cases (see example in section 5), the essential support of $d$ at $P$ is known to be composed of only $C_{+}(P)$ and $C_{-}(P)$. Hence, if it is shown that the essential support of $r$ does not contain $C_{+}(P)$, this will be sufficient to ensure that it is (contained in) $C_{-}(P)$ (since $r=f-d$ ).

The assumption of mixed- $\alpha$ cancellation can be restricted correspondingly.

ii) No assumption would be necessary if mixed- $\alpha$ Landau diagrams $\mathscr{D}_{B}$ were not involved at $P$, or in the cases of remark i), if the relative configurations of their external trajectories could not correspond to $C_{+}(P)$.

We shall see however on the example of section 5 that $C_{+}(P)$ is expected in general to be in the essential support of some functions $f_{B}$ involved in $r$, (because of some mixed- $\alpha$ diagrams).

b) More general "discontinuity formulae"

The discussion of paragraph a) has excluded cases when $P$ lies on the closure of several $+\alpha$-Landau surfaces. In such cases, the term $d$ associated with a given graph $D$ involved at $P$ cannot be expected in general to be a discontinuity of $f$ in the sense of paragraph $a$ ).

If $D$ is a "parent graph" involved at $P$ (i.e. lies on the $+\alpha$-Landau surfaces of one or several graphs which are contrations of $D$ and lies in the closure of $L\left(D_{+}\right)$), then one is led to introduce a term $\hat{d}$ which is analogous to $d$, but is obtained by replacing each vertex of $D$ by the kernel of the $S$ matrix itself, rather than by its connected part.

General results which cover such cases follow from the work of Coster and Stapp cited in [8], with assumptions similar to above. They can be stated by saying that the essential support at $P$ of $s-d$ does not contain appropriate directions ( $s$ is the non connected kernel of the $S$ matrix, after factorization of $\delta^{4}\left(\sum p_{i}-\sum p_{j}\right)$ : see section 1$)$.

c) Physical significance of discontinuity formulae [10].

The discontinuity formulae discussed in paragraphs a) and b) have in usual cases a very satisfactory physical interpretation.

Consider, as in section 2 , a set of initial and final wave functions of the form (7). Macrocausality says that there is exponential fall of the connected amplitudes between these wave functions if $(P, u)$ is non-causal, 
but gives no information in the opposite case, when $(P, u)$ is causal. The discontinuity formula of paragraph $a$ ) ensures that $C_{+}(P)$ does not belong to the essential support at $P$ of $r=f-d$. It therefore says that the connected amplitudes $S^{c}\left(\left\{\left(\phi_{k}\right)_{\tau, u_{k}, P_{k}}\right\}\right)$ is then equal, up to a remainder which has an appropriate exponential decrease in the $\tau \rightarrow \infty$ limit, to the integral, over internal on-mass-shell 4 -momenta of $D$, of the product of scattering amplitudes associated with each vertex of $D$. i.e. there is a factorization (up to exponential fall off) corresponding precisely to the classical multiple scattering diagram $\mathscr{D}$ involved at $P$.

For graphs $D$ with sets of multiple lines, the physical interpretation is somewhat more subtle, but still satisfactory.

The same type of conclusions holds in the situations of paragraph $b$ ).

Finally, in usual cases, there is moreover equivalence between discontinuity formulae and the above mentioned factorization properties of scattering amplitudes for causal configurations.

\section{§5. The Pole-Factorization Theorem [11]}

In this section we consider the following graph $D$ :

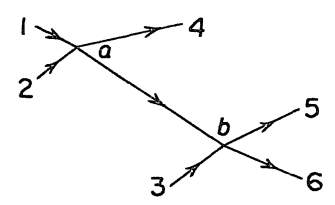

and we shall assume that $\left(P_{1}+P_{2}+P_{3}\right)^{2}<16 \mathrm{~m}^{2}$.

The causal diagram $\mathscr{D}$ at $P$, whose relative configuration of external trajectories is $C_{+}(P)$ is by definition (see section 2) such that $1,2,4$, resp. 3, 5, 6 meet at a space-time representative point $A$ of $a$, resp. $B$ of $b$, with $A B=\lambda K, \lambda>0, K=P_{1}+P_{2}-P_{4}\left(=P_{5}+P_{6}-P_{3}\right)$.

a) Decomposition of the form (10)

By using the developments of $S$ and $S^{-1}$ into connected parts, the equation $S S^{-1}=1$ can be written (below the 4-particle threshold) in the form: 


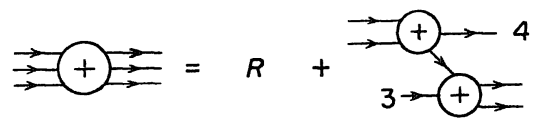

where

$$
\begin{aligned}
& R=H+H \overrightarrow{\Rightarrow \oplus}^{4}
\end{aligned}
$$

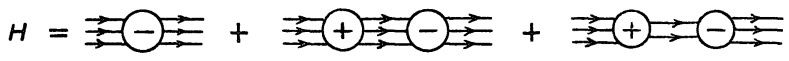

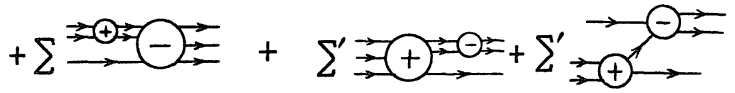

In these equations, $\Rightarrow-\rightarrow$ represents minus the connected kernel of $S^{-1}$, according to a usual convention. The sums $\sum$, resp. $\Sigma^{\prime}$, in (14) refer to the various ways of assigning sets of external (initial or final) 4-momenta to each bubble, resp. corresponding sums from which the term $\stackrel{\rightarrow}{\rightarrow} \rightarrow-4$, or $\overrightarrow{3 \longrightarrow C} \rightarrow$ by definition, the kernel of $\quad \overrightarrow{\rightarrow \rightarrow(4)}$ is (with notations similar to above).

A simple examination of (12) shows that it is (after factorization to $\delta^{4}\left(\sum p_{i}-\sum p_{j}\right)$ a decomposition of $f$ of the form (10) where $d$ and $r$ have the properties i) and ii) $\left(R=r \times \delta^{4}\left(\sum p_{i}-\sum p_{j}\right)\right.$,

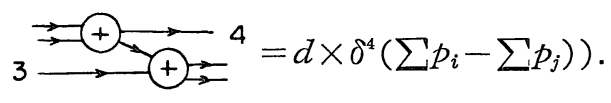

If the assumption of mixed- $\alpha$ cancellation is used, then $d$ appears, as explained in section $4 \mathrm{a}$ ) as the discontinuity of $f$, and hence, in this particular case, $f$ can be locally written in the form:

$$
f=\frac{1}{2 i \pi} \frac{a\left(p_{1} \cdots p_{6}\right)}{k^{2}-m^{2}+i \varepsilon}
$$

where $k=p_{1}+p_{2}-p_{4}$ and $a$ is a locally analytic function such that:

$$
\left.a\left(p_{1} \cdots p_{6}\right)\right|_{k=m_{2}}=f_{2,2}\left(p_{1}, p_{2} ; p_{4}, k\right) f_{2,2}\left(p_{3}, k ; p_{5}, p_{6}\right)
$$

The set of relations (15) (16) is called the pole-factorization theorem. (Similar results hold in the case of subsection $4 \mathrm{~b}$ )). 
b) Examination of mixed- $\alpha$ diagrams.

The essential support of $d$ at $P$ is composed only, as easily checked, of $C_{+}(P)$ and $C_{-}(P)$. According to remark i) at the end of section 4a), we first wish to check if there are at $P$ mixed- $\alpha$ diagrams $\mathscr{D}_{B}$ (for some of the functions $f_{B}$ involved in $r$ ) whose relative configuration of external trajectories is $C_{+}(P)$. If not, the discontinuity formula would be proved without need of any extra assumption and without extra work: see remark ii) at the end of section $4 a$ ). The detailed study shows that this hope is completely unjustified.

The complete study of all possible mixed- $\alpha$ diagrams is too long to be presented here, and we shall only describe the main facts. The term $R$ in (12) is the sum of $H$ and of $H \rightarrow \longrightarrow \rightarrow 4$. We first examine $H$ in paragraphs i), ii), iii) and give in iv) an example of a further difficulty arising from the "multiplication" of $H$ by $\underset{\rightarrow \rightarrow \longrightarrow}{\longrightarrow}$.

i) Consider the term 3 that $P$ is always a " $u=0$ " point (associated with a mixed- $\alpha \mathscr{D}_{B}$ which has been described). Hence, all directions, including $C_{+}(P)$, can a priori be singular at $P$ (on the basis of macrocausality and unitarity alone).

Throughout the remainder of this text, we shall admit the regularity conjecture at $u=0$ points stated at the end of section 3 , in which case the present difficulty is removed.

ii) Other difficulties occur however for the same term

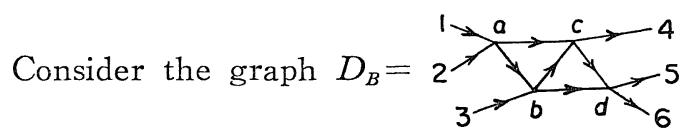

and possible space-time representation $\mathscr{D}_{B}$, if they exist, in which the line $b \quad d$ has zero length.

Let us define a subset $\Omega_{+}$of $L\left(D_{+}\right)$as follows. Being given $p \in$ $L\left(D_{+}\right)$, we choose a point $A$ in space-time, draw through it two lines respectively parallel to $p_{4}$ and $k=p_{1}+p_{2}-p_{4}$, and choose $B$ on the second one, with $(A B)_{0}>0$. Then $p \in \Omega_{+}$if it is possible to find two on massshell 4-momenta $k_{1}, k_{2}\left(k_{i}{ }^{2}=m^{2}, \quad k_{i_{0}}>0, i=1,2\right)$ such that $k_{1}+k_{2}=k+p_{3}$ $\left(=p_{5}+p_{6}\right)$ and such that the line parallel to $k_{1}$ and passing through $B$ 
meets the line passing through $A$ and parallel to $p_{4}$ at a point $C$ such that $(A C)_{0}>0,(B C)_{0}>0$ :

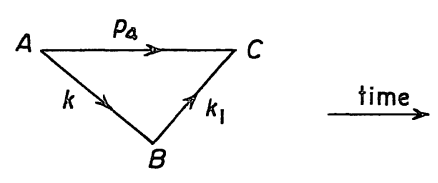

If (and only if) $p \in \Omega_{+}$, one checks easily that there exists a $\mathscr{D}_{B}$ at $p$ and that its relative configuration of external trajectories corresponds to $C_{+}(p)$.

An elementary analysis shows that $\Omega_{+}$is a full open subset of $L\left(D_{+}\right)$.

iii) In a number of cases, $C_{+}(P)$ appears as a possible singular direction of certain $f_{B}$, associated with mixed- $\alpha$ diagrams, but only when $P$ lies on certain lower dimensional subsets of $L\left(D_{+}\right)$.

Consider for instance the term

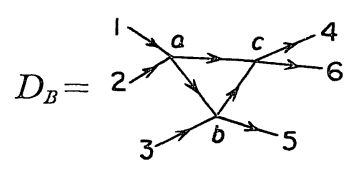

One checks easily that there is a $\mathscr{D}_{B}$ corresponding to $C_{+}(P)$ if for instance $p_{3}=p_{5}$. The line $a c$ has then zero length $(A=C)$. Hence $1,2,4$ pass through $A$. Since $p_{6}=p_{1}+p_{2}-p_{4}$, the trajectory of line 6 meets those of 3,5 at $B$ and $A B$ is directed along $p_{1}+p_{2}-p_{4}$.

iv) Although the situations of paragraph iii) occur only for points $p$ which lie in lower dimensional subsets of $L\left(D_{+}\right)$, they may lead, after "multiplication" of $H$ by $\underset{\longrightarrow}{\longrightarrow}$ to cases when $C_{+}(P)$ will be a possible singular directions at all points of $L\left(D_{+}\right)$.

Consider for instance the term

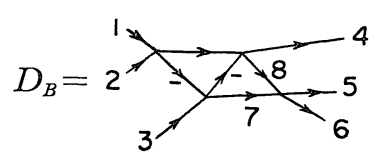

The above mentioned fact arises from the possibility of putting $p_{7}=p_{3}$ in the subdiagram associated with 
described in paragraph iii), but there is now no constraint on the actual external 4-momenta of the complete bubble diagram function.

c) New derivation of the pole factorization theorem.

The more refined proof of the pole factorization that we briefly outline now does not use the mixed- $\alpha$ cancellation assumption, but uses instead two weak regularity conjectures on scattering and bubble diagram functions. The first one is the conjecture already mentioned on $u=0$ points. The second one is a slight refinement of the analyticity properties associated with macrocausality. We describe it below for simplicity in a form which is slightly stronger than that really needed and at points $p$ which lie on no $+\alpha$-Landau surface other than $L\left(D_{+}\right)$. Otherwise, the conjecture applies to the appropriate contribution to $f$ which appears when the decomposition theorems of essential support, or singular spectrum, theory are used.

Macrocausality entails that $f$ is at $p$ the boundary value of an analytic $f$ from the "plus $i \varepsilon$ " directions of the open half-space dual to $C_{+}(p)$. Let $\phi$ be a real analytic function such that $L\left(D_{+}\right)$is represented locally in $\mathscr{M}$ by the equation $\phi=0$, and chosen such that $\phi>0$ on the physical side of $L\left(D_{+}\right)$(for instance, in the case under study, $\phi=k^{2}-m^{2}$ ). If $\phi$ is one of a system of real analytic local coordinates of $\mathcal{M}$ around $p$, the open half space dual to $C_{+}(p)$ is represented in this system by $\mathrm{Im}$ $\phi>0$ (in the space of imaginary parts) and the above property of $f$ means that being given any open cone $\Gamma$ with apex at the origin whose closure is contained (apart from the origin) in this open half-space, there exists a complex neighbourhood $\omega$ of $p$ such that all points in $\omega \cap\{\operatorname{Im} \phi \in \Gamma\}$ belong to the analyticity domain of $f$. We then state:

\section{Regularity conjecture on $f$}

" $f$ is moreover analytic in a domain of the form $\omega \cap\{\operatorname{Im} \phi>0\}$ (where $\omega$ is a given complex neighbourhood of $p$ )."

This conjecture (and its extension to cases when $p$ lies on other $+\alpha$-Landau surfaces) first allows one to remove the problems of paragraph iii) of subsection $\mathrm{b}$ ) in the study of $h$, i.e. to show that $C_{+}(p)$ 
is not a singular direction of $h$ at points $p$ which do not lie in $\Omega_{+}$. (To prove this result, one uses the fact that $H=\Rightarrow+(+\infty)-$

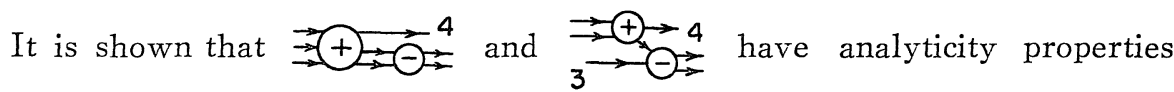
analogous to those of $\underset{\rightarrow}{\rightarrow}$ itself, and Bremerman's continuity theorem is then used to exclude "isolated" singularities of $h$ along subsets of $L\left(D_{+}\right)$ of codimension larger than one.) The above result is then extended from $h$ to $r$. Finally, the same conjecture as above (or alternatively a refined use of unitarity) entails that $C_{+}(p)$ is not a singular direction of $r$ even at $\Omega_{+}$points. (The fact that $R=\rightarrow+\rightarrow \Rightarrow$ at this stage.) Note the result is not proved, and not expected to be true, for $H$ itself.

\section{§ 6. Conclusion}

The discontinuity formulae are certainly believed to be satisfed by the actual $S$-matrix, on the basis of many arguments. Is it however possible to derive them on the basis only of physical principles, such as macrocausality and unitarity? The latter implies strong links between various multiparticle amplitudes which maybe have not yet been fully exploited, and the results of section 5 are a step in this direction, even though two (weak) regularity conjectures have been used, besides macrocausality and unitarity.

A first program would be to extend these latter results to more general cases, and to determine what are the minimal and mathematically neat conjectures needed.

The work accounted in section 5 gives the hope that it might be possible to introduce a slightly refined notion of essential support, or singular spectrum, which would contain a small information on the nature of singularities (and not only on the location of singular directions), and would possibly allow one to prove all needed results from a slightly refined statement of macrocausality.

One would then hope also to have a satisfactory physical interpretation of such a statement. It would probably have to contain some infor- 
mation in the causal directions. Hopefully this information will be very limited. In fact, we already know (see section 4c)) that the factorization properties for causal configurations are equivalent and hence do imply the discontinuity formulae, in a number of cases. However, our feeling (confirmed by section 5) is that much less is needed; i.e. the discontinuity formulae should be derived "essentially" from macrocausality and unitarity, and they in turn imply the factorization properties for causal configurations.

Another approach to these problems would be to study if a weak form of the conjecture by Professor Sato (according to which the $S$-matrix should be holomonic) would allow one to derive the discontinuity formulae. The strong form of Sato's conjecture, which includes information on orders, etc.. is probably too strong for our present purpose, and we prefer to consider it as a consequence of the discontinuity formulae, as has been proved already in various cases:see the lecture by Professor Kawai.

\section{References}

[1] Iagolnitzer, D., The S-matrix, North-Holland, Amsterdam, to be published, or the earlier version of that work entitled Introduction to S-matrix theory, ADT, Paris (1973).

[2] Iagolnitzer, D. and Stapp, H. P., Comm. Math. Phys., 14 (1969), 15.

[3] Bros, J. and Iagolnitzer, D., exposés 16, 18 in Proceedings of the 1974/1975 Goulaouic-Lions-Schwartz Séminaires.

Iagolnitzer, D., in Structural Analysis of Collision Amplitudes, Part III, ed. by Balian, R. and Iagolnitzer, D., North-Holland, Amsterdam (1976).

[4] Sato, M., Kawaï, T. and Kashiwara, M. in Hyperfunctions and Pseudo-Differential Equations, Springer Verlag, Heidelberg (1973).

[5] The presentation of this section follows ch. II of references 1 which is an improvement, in terms of essential support, of [2]. The elaboration of the subject has also involved basic works by other authors, in particular

Omnès, R., Phys. Rev. 146 (1966), 1123.

Chandler, C. and Stapp, H. P., J. Math. Phys. 90 (1969). 826.

Pham, F., Ann. Inst. Poincaré, Vol. 6, n 2 (1967), 89.

[6] Pham, F., in Hyperfunctions and Theoretical Physics, Springer-Verlag, Heidelberg (1975).

[7] The presentation of the structure theorem given here is that of Iagolnitzer, D., Comm. Math. Phys. 41 (1975), 39. For earlier results on the subject, in somewhat different (and weaker) forms, see Stapp. H. P., J. Math. Phys. 9 (1968), 1548. See also References 8 and 9.

[8] The presentation given here is essentially that of Coster, J. and Stapp. H. P., J. Math. Phys. 10 (1969), 371, 11 (1969), 2743. See related results in: 
[9] Bloxham, M. I. W., Olive, P. I., Polkinghorne, J. C., J. Math. Phys. 10 (1969), 494, 10 (1969), 545, 10 (1969), 553.

[10] See details in [1] and references quoted therein, in particular Pham, F., Op. Cit. in reference 5 and Iagolnitzer, D., in Lectures in Theoretical Physics, ed. by Mahanthappa, K. T. and Brittin, W. E., Gordon and Breach, New York (1969).

[11] The pole-factorization theorem was first "proved" by Olive, D., in 1964 with implicit assumptions (which include at least mixed- $\alpha$ cancellation). The new proof described in sections $5 b$ ) and $5 c$ ) is due to Iagolnitzer, D. and Stapp. H. P., in preparation. 